

\title{
COUPLED FLOW, THERMAL AND STRUCTURAL ANALYSIS \\ OF AERODYNAMICALLY HEATED PANELS
}

\author{
By \\ Earl A. Thornton ${ }^{1}$ and Pramote Dechaumphai ${ }^{2}$
}

\section{SUMMARY}

The following paper will be presented at the April 6-8, 1987, meeting of the AIAA/ASME/ASCE/AHS 28th Structures, Structural Dynamics and Materials Conference. The paper describes research work supported under NASA/Langley Research Grant NSG 1321, and is submitted to fulfill the progress report requirement on the grant, for the period ended December 31, 1986.

1 Professor, Department of Mechanical Engineering and Mechanics, 01d Dominion University, Norfolk, Virginia 23508.

2 Research Associate, Department of Mechanical Engineering and Mechanics, $01 d$ Dominion University, Norfolk, Virginia 23508. 


\author{
Earl A. Thornton* and Pramote Dechaumphai ${ }^{* *}$ \\ old Dominion University \\ Norfolk, Virginia
}

\section{Abstract}

A finite element approach for coupling flow, thermal and structural analyses of aerodynamically heated panels is presented. The NavierStokes equations for laminar compressible flow are solved together with the energy equation and quasi-static structural equations of the panel. Interactions between the flow, panel heat transfer and deformations are studied for thin stainless steel panels derodynamically heated by Mach 6.6 flow.

\section{Momenclature}

$\begin{array}{ll}A & \text { element area } \\ c & \text { fictitious damping constant } \\ C_{v} & \text { specific heat at constant volume } \\ E, F & \text { flux components in } x \text { and } y \\ & \text { directions } \\ E_{t} & \text { fluid total energy } \\ {[M]} & \text { mass matrix } \\ {[N(x, y)]} & \text { element interpolation functions } \\ p & \text { fluid pressure } \\ q_{x}, q_{y} & \text { heat fluxes } \\ \{R\} & \text { load vector } \\ T & \text { temperature } \\ t & \text { time } \\ \Delta t & \text { time step } \\ U & \text { typical conservation variable }\end{array}$

$u, v$ flow velocity components, eq. (2) or solid displacement components, eq. (4)

$\begin{array}{ll}x, y & \begin{array}{l}\text { coordinate direct } \\ \rho\end{array} \\ \sigma_{x x} \cdot \tau_{x y}, \sigma_{y y} & \text { fluid stress comp } \\ \sigma_{x}, \tau_{x y}, \sigma_{y} & \text { solid stress conpo } \\ \text { Subscripts } & \\ I & \text { inviscid flux } \\ V & \text { viscous flux } \\ n & \text { time step index }\end{array}$

Superscript

$n \quad$ time step index

\footnotetext{
* Professor, Department of Mechanical Engineering and Mechanics, Senior Member AIAA

** Research Associate, Department of Mechanical Engineering and Mechanics, Member AIAA
}

\section{Introduction}

Thermal-structural analysis me thods have an important role in the design of high speed flight vehicles that experience severe derodynamic heating. Traditionally the prediction of thermally induced deformations and stresses has been accomplished as a sequence of analyses. First, heating rates are predicted on aerodynamic surfaces using either empirical methods or computationally using finite difference methods. Then, the structural temperatures are determined using a network-type thermal analysis or more recentiy via a finite element thermal analysis. Finally, structural deformations and stresses are computed by a finite element analysis using temperatures as input data. This approach is generally effective and indeed has been used routinely in the design of flight vehicles exposed to severe thermal environments. However, there are two recognized shortcomings of the approach: (1) the sequence of analyses is relatively inefficient because the incompatible models used in the three analyses make data transfer difficult, and (2) the approach assumes that the heat transfer between the fluid and structure and thermally induced deformations have negligble effects on the aerodynamic heating. The effects of the heat transfer and deformations on the heating may be considered by an iterative sequence of analyses, but the process is cumbersome and usually is not a ttemped.

Yet there exist inportant design problems where fluid-thermal-structural interactions are important. One example are metallic thermal protection systems tested in the Mach 7 8-foot high temperatures tunnel (HTT) at the NASA Langley Research Centerl. The tests show that panels "bowed-up" into the flow to produce heating rates that are up to 1.5 times rates based on flat plate predictions. Aerothermal loads on spherical dome protuberances have been studied both computationally ${ }^{2}$ and experimentally $y^{3}$. The computations and experiments show that heating rates are augmented on windward surfaces, and that the increase in heating rates depend on the protuberance height compared to the boundary layer thickness. The computational and experimental determination of the augmented heating rates were based on assumed surface configurations and neglect flow-structural deformation interactions. A second example of important problems where flow, thermal, and structural interactions are important is the scramjet engine structure for the national aerospace plane. Figure 1 shows the sidewalls and leading edge of the engine structure where interactions may be significant. The leading edge of the internally cooled scramjet fuel injection strut is an especially critical area. 
Research is underway at the NASA Langley Research Center to improve the capabilities and efficiency of finite element high speed compressible flow analysis methods and to develop efficient coupling of finite element fluid, thermal and structural analyses. The focus of the research is the prediction of aerothermal loads as well as the thermal-structural response of complex three dimensional bodies. The research combines analysis with experimental studies conducted in the $8^{\prime}$ HTT at NASA Langley.

The purpose of this paper is to describe research in the coupling of flow, thermal and structural analyses by the finite element method for aerodynamically heated panels. The study of the interactions for panels is a preliminary, but important, step towards the objectives of analyzing more realistic structures such as thermal protection systems and scramjet engine structures. The paper will bring together for the first time the solution of the Navier-Stokes equations for the prediction of aerodynamic heating and the solution for the associated thermal-structural equations by a single finite element algorithm in one integrated, vectorized program. The authors and co-workers have developed the methodology to solve the separate problems over the last few years with this goal in mind. Initial progress in solving inviscid compressible flows by the finite element method is described in recent papers ${ }^{4-5}$. Progress in solving viscous compressible flows is described in reference ${ }^{6}$. The application of the CFD algorithm to thermal-structural problems is described in a paper ${ }^{7}$ presented at 27 th SDM conference in San Antonio, Texas.

The problem formulation will be described first, then the solution approach will be presented. The Taylor-Galerkin algorithm will be highlighted, and the solution sequence for the coupled problem is presented. Finally, numerical results from two coupled flow-panel interactions are presented and discussed.

\section{Flow-Thermal-Structural Formulation}

The fluid flow is described by the conservation of mass, momentum and energy equations for a laminar flow of a perfect gas assuming temperature dependent viscosity and thermal conductivity. The thermal behavior of the panel is described by the conservation of energy equation, and the structural behavior is described by the quasi-static equations of motion for a solid. Radiation heat transfer from the panel to space is considered assuming the fluid is perfectly transparent. Thermal properties such as specific heat and thermal conductivity are assumed to temperature dependent. Largestrain displacement relations are used for the panel to permit large deformations, and nonlinear, temperature-dependent stress-strain relations are employed to permit plastic deformation. The equations for the fluid and solid are written in conservation form.
Fluid

$$
\frac{\partial\{U\}}{\partial t}+\frac{\partial\left\{E_{I}-E_{V}\right\}}{\partial x}+\frac{\partial\left(F_{I}-F_{V}\right\}}{\partial y}=0
$$

where $\{U\}$ is a vector of the conservation variables for the fluid, $\left\{E_{I}\right\}$ and $\left\{F_{I}\right\}$ are inviscid flux components, and $\left\{E_{v}\right\}$ and $\left\{F_{v}\right\}$ are viscous flux components. These vectors are given by

$$
\begin{aligned}
\{U)^{\top} & =\left[\begin{array}{llll}
\rho & \rho u & \rho v & \rho E_{t}
\end{array}\right] \\
\left\{E_{I}\right\}^{\top} & =\left[\begin{array}{lllll}
\rho u & \rho u^{2}+p & \rho u v & \left(\rho E_{t}+p\right) u
\end{array}\right] \\
\left(F_{I}\right\}^{\top} & =\left[\begin{array}{llll}
\rho v & \rho v u & \rho v^{2}+p & \left(\rho E_{t}+p\right) v
\end{array}\right] \\
\left\{E_{v}\right\}^{\top} & =\left[\begin{array}{llll}
0 & \sigma_{x x} & \tau_{x y} & \left(u \sigma_{x x}+v \tau_{x y}-q_{x}\right)
\end{array}\right] \\
\left\{F_{V}\right\}^{\top} & =\left[\begin{array}{llll}
0 & \tau_{x y} & \sigma_{y y} & \left(u \tau_{x y}+v \sigma_{y y}-q_{y}\right)
\end{array}\right]
\end{aligned}
$$

where $\rho$ is the fluid density, $u$ and $v$ are velocity components, $E_{t}$ is the total energy, $\sigma_{x x}, \sigma_{y y}$ and $\tau_{x y}$ are viscous stress components, and $q_{x}, q_{y}$ are heat fluxes. In the inviscid flux components, the pressure $p$ is related to the total energy for a perfect gas assuming a constant ratio of specific heats. The stress components are related to the velocity gradients assuming the Stokes' hypothesis, and the heat fluxes are related to the temperature gradients by Fourier's law. The temperature dependent viscosity is computed from Sutherland's law, and the thermal conductivity is computed assuming a Prandtl number equal to 0.72 .

Solid

$$
\frac{\partial\{U\}}{\partial t}+\frac{\partial\{E\}}{\partial x}+\frac{\partial\{F\}}{\partial y}=0
$$

where $\{U\}$ is a vector of the conservation variables for the solid; $\{E\}$ and $\{F\}$ are "flux" components for the solid. These vectors are given by

$$
\begin{aligned}
& \{U\}^{\top}=\left[\begin{array}{lll}
c u & c v & \rho c_{v} T
\end{array}\right] \\
& \{E\}^{\top}=\left[\begin{array}{lll}
-\sigma_{x} & -\tau_{x y} & q_{x}
\end{array}\right] \\
& \{F\}^{\top}=\left[\begin{array}{lll}
-\tau_{x y} & -\sigma_{y} & q_{y}
\end{array}\right]
\end{aligned}
$$

where $u$ and $v$ are displacement components, $p$ is the solid density, $c_{v}$ is the specific heat, and $T$ is the temperature. The coefficient $c$ of the displacement components is a fictitious damping constant that is used to facilitate time marching to a steady-state quasi-static solution. The solid's stress components $\sigma_{x}, \tau_{x y}$ and $\sigma_{y}$ are reiated to the displacement gradients within the elastic range using generalized Hooke's law. In the plastic range, a modified elastic stiffness based upon an effective strain is employed using 
the material's non-linear uniaxial stress-strain curve $^{8}$. The solid's heat fluxes $q_{x}$ and $q_{y}$ are related to the temperature gradients by Fourier's law.

The sets of equations (1) and (3) are solved subject to appropriate boundary, interface and initial conditions for the fluid and solid. Flow boundary conditions correspond to supersonic flow hence all conservation variables are specified on in-flow surfaces. On supersonic outflow surfaces, the finite element formulation provides appropriate natural boundary conditions. Solid boundary conditions include either specified displacments or surface tractions. At the fluidsolid interface, flow velocity components are zero, but the fluid density as well as the fluidsolid temperature are unknown. Continuity of temperature at the fluid solid interface is imposed explicitly in the solution algorithm. A heat flux energy balance is imposed at the fluidsolid interface to account for the fluid-solid conduction heat fluxes and the emitted radiation heat flux. Initially, the flow is assumed uniform at free stream values; the solid is assumed undeformed and unstressed at room tempera ture.

\section{Solution Approach}

The solution approach solves equations (1) and (3) using an explicit time marching scheme, the Taylor-Galerkin algorithm, that has been described in previous papers. ${ }^{4-7}$ Herein, the essential features of the algorithm are highlighted, and the solution sequence for the coupled problem is presented.

\section{Tayior-Gaierkin Aigorithm}

The basic concept of the Taylor-Galerkin algorithm is to use: (1) Taylor series expansions in time to establish recurrence relations for time marching, and (2) the method of weighted residuals with Galerkin's criteria to develop the finite element matrix equations describing the spatial distribution of the dependent variables.

For simplicity, the finite element formulation will be given for a single scalar equation,

$$
\frac{\partial U}{\partial t}+\frac{\partial E}{\partial x}+\frac{\partial F}{\partial y}=0
$$

where the variables $U, E$ and $F$ are analogous to the corresponding vector quantities in eqs. (1) or (3). Let $\{U\}^{n}$ denote the element nodal values of the flow variables $U(x, y, t)$ at time $t_{n}$. The time step $\Delta t$ spans two typical time $t_{n}$ and $t_{n+1}$ in the transient response. The computation proceeds through two time levels. $t_{n+1 / 2}$ and $t_{n+1}$. At time level $t_{n+1 / 2}$, values for $u$ that are constant within each element are computed explicitly. At time level $t_{n+1}$, the constant element values computed at the first time level are used to compute nodal values for $U$. In the time level $t_{n+1}$ computations, element contributions are assembled to yield the global equations for nodal unknowns. The "mass" matrix for the resulting equations is approximately diagonalized to yield an explicit algorithm.

Time Level $t_{n+1 / 2}$

The constant element value $U_{0}^{n+1 / 2}$ is computed from

$$
\begin{aligned}
A U_{D}^{n+1 / 2} & =\int_{A}[N] d A\{U\}^{n}-\frac{\Delta t}{2} \int_{A}\left[\frac{\partial N}{\partial X}\right] d A\{E\}^{n} \\
& -\frac{\Delta t}{2} \int_{A}\left[\frac{\partial N}{\partial y}\right] d A\{F\}^{n}
\end{aligned}
$$

where $A$ denotes an element's area, $\Delta t$ is the time step and $[N]$ is a matrix of interpolatign functions. Quantities in braces, such as $\{E\}^{n}$, represents nodal values at time $t_{n}$.

Time Level $t_{n+1}$

The nodal values for a single element are given by

$$
\begin{aligned}
{[M]\{U\}^{n+1} } & =[M]\{U\}^{n}+\Delta t \int_{A}\left\{\frac{\partial N}{\partial x}\right\} d A E\left(t_{n+1 / 2}\right) \\
& +\Delta t \int_{A}\left(\frac{\partial N}{\partial y}\right\} d A F\left(t_{n+1 / 2}\right)+\{R\}^{n+1 / 2}
\end{aligned}
$$

where $\{R\}^{n+1 / 2}$ represents known boundary terms. The matrix [M] is the consistent mass matrix; to produce an explicit algorithm, $[M]$ is diagonalized. Following usual finite element procedures, the element matrices given in equation (7) are then assembled to form system equations.

The two time level Taylor-Galerkin algorithm is conditionally stable, and the time step for the fluid flow must satisfy stability requirements based on the CFL condition and the Reynolds number. Artificial viscosity, the form due to Lapidus, is used to reduce oscillations in the flow computations.

The time evolution of the flow and the solid thermal response is computed in a "time-accurate" fashion using the algorithm as described by tine marching to steady-state via a viscous relaxation procedure based on the Taylor-Galerkin algorithm. ${ }^{7}$ To speed convergence of the quasistatic solution to steady-state, the concept of accelerated viscous relaxation is employed. ${ }^{9}$

\section{Solution Sequence}

Experimental and computational results for flows over panels show that the flow initially approaches steady-state in a much shorter time than required for the panel to respond thermally and structurally. Typically, heating rates approach steady-state in about a milli-second. A pane1 at this time remains virtually undeformed 
at temperatures only slightly higher than the initial temperature. After a few seconds, panel temperatures begin to rise appreciably and significant deformations occur. During this time, thermal and deformation coupling effects alter the flow field. The coupling effects continue to alter the flow and panel behavior until the panel reaches thermal equilibrium. Typically, panel thermal equilibrium is approached after 5 to 10 minutes of aerodynamic heating.

Based on this sequence of events, the solution sequence shown in Figure 2 has been adopted. The solution sequence alternates between coupled flow and thermal analyses of the fluid/panel and thermal analysis of the panel. At selected times, temperatures of the panel are used to compute structural deformations. If significant, the structural deformations are used to define a new flow boundary.

\section{Applications}

Two applications are presented to illustrate the computational approach and investigate the coupled response of aerodynamically heated panels. The first application is a "flat" panel that in the undeformed state has zero angle of attack. The second application is a panel that in the undeformed state is oriented at 5 degrees to the flow so that initially it forms a compression corner.

The flow conditions for the two applications correspond to test conditions in the 8 foot HIT at NASA Langley. A schematic of a proposed experiment to validate the flow-thermalstructural interaction analysis is shown in Fig. 3. In the proposed experiment, test panels are mounted in a panel holder with a sharp leading edge. The panel holder is oriented at 15 degrees to the tunnel free stream so that an oblique leauting edge shock forms producing the desired boundary layer on the panel holder. The figure schematically shows a panel supported in the panel holder in a initial flat orientation being heated at time $t=0$ by the boundary layer flow. After some time, the test panel deforms into the stream altering the flow significantly by introducing local shocks, expansion regions and shock-boundary and layer interactions.

\section{Flat Panel}

The finite element model and boundary conditions for the flat panel are shown in Fig. 4. The test panel is 4 inches long, has a thickness of 0.1 inch and is made from $A M-350$ stainless steel. The flow conditions and profiles at the left boundary of the computational domain were determined from a boundary layer solution for the panel holder shown in Fig. 3. The computational domain is modeled with a finite element mesh of 5285 nodes and 5120 quadrilaterial elements. About $95 \%$ of the nodes lie in the flow domain. The mesh is graduated normal to the panel to produce high resolution of flow variables at the flow-solid interface. The black band next to the panel in Fig. 4 indicates the high concentration of elements in the boundary layer near the panel. About 10 nodes in the vertical direction lie within the boundary layer. At the left and right ends of the panel, conduction heat transfer is permitted to the panel holder. The bottom surface of the panel is assumed perfectly insula ted.

The two cases of panel structural boundary conditions considered are shown in Fig. 4. In the first case, the panel is supported by immovable supports on the bottom corners as shown. For these boundary conditions, the panel deforms into a convex shape. In the second case, the panel is supported by immovable supports at the top corners. For these boundary conditions, the panel deforms into a concave shape.

The flow-thermal-structural interaction for the flat plate was analyzed using the solution sequence shown in Fig. 5. The figure shows that panel deformations were computed three times at ten second intervals for a test duration of 30 seconds. Calculations were also performed where the panel deformations were computed only once after a heating interval equal to the total test duration of 30 seconds. For convex deformations, the results for the two analysis sequences were in excellent agreement, but for the concave deformations the results indicate that a smaller interval for deformation updates is required. The results to be presented in subsequent figures for the convex and concave deformations of the flat panel are for the analysis sequence shown in Fig. 5, i.e. three computations of panel de forma tions.

In a typical coupled flow-thermal analysis ( $F / T$ in $F i g .5)$, a time step of $1 . E-7 \mathrm{~s}$ was used. and 4000 steps were required to approach steady heating rates in about $0.4 \mathrm{milli-second.} \mathrm{In} \mathrm{a}$ typical thermal analysis ( $T$ in Fig. 5), a time step of $0.01 \mathrm{~s}$ was used, and $1000 \mathrm{steps}$ were required to heat the panel for ten seconds. A test duration of $30 \mathrm{~s}$ is early in the transient response of the panel; radiation equilibrium is estimated to occur after about 600-900s of hea ting.

The interaction between the panel deformation and the flow density distribution is shown in Fig. 6 at three times, $t=10,20,30 \mathrm{~s}$. The figure shows the development of a shock eminating from the left support on the windward side of the deformed panel. The fluid density increases through the shock, but the density decreases as the flow expands across the convex center of the panel along the leeward side towards the right support. As the flow is turned by the panel near the outflow, a recompression occurs and the density begins to increase as a recompression shock is developed. As the panel deforms, the boundary layer thickness is altered over the panel becoming appreciably thicker on the leeward surface of the panel and thicker on the panel holder near the outflow.

The evolution of the temperature at the fluid-panel interface is presented in Fig. 7 for the panel with convex deformation. Due to the small thickness of the panel, there is virtually no temperature gradient through the panel thickness, and the panel temperature varies only with $x$ as shown. The "rounding" of the temperature distributions near the left and right panel 
supports is due to conduction heat transfer to the panel holder.

The heating rate for the panel with convex deformation is presented in Fig. 8. Figure 8a shows the evolution of the heating rate distribution as the panel deforms for $t=0,10,20,30$ s. The heating rate distribution displays the effects of both the fluid-panel heat transfer and the panel deformation. The overall decrease in the the heating rate at each time is due to the rise of the panel temperature. Fluid temperatures (not shown) near the panel at each time remain roughly the same, and due to the rise of the panel temperature there is a lower temperature gradient at the fluid-panel interface that produces the lower heating rate. The panel deformation causes the increase in the heating rate distribution on the windward side of the panel for $1<x<2$.

The effect of the panel temperature on heating rate distribution is illustrated further in Fig. 8b. This figure compares the heating rate of a deformed panel assuming a constant specified temperature to the heating rate that occurs when the panel temperature is perinitted to rise as in Fig. 7. The upper curve for the specified panel temperature shows the significant rise in heating rate for $1<x<2$ clearly demonstrating the deformation coupling effect.

The evolution of the thermal stress distributions on the top and bottom surfaces of the panel is presented in Fig. 9 for the convex deformation case. The panel experiences a large compression stress because longitudinal expansion is prohibited by the immovable supports. Because the supports are offset from the panel neutral surface, bending is superimposed that produces small tensile stresses on the panel's top surface. The local oscillations in the bottom surface stresses at $x=1$ and $x=5$ are unrealistic and indicate the need for further mesh refinement in these areas.

The second case considered the supports at the top corners of the panel producing concave deformation. The flow density distribution at $t=30 \mathrm{~s}$ for the panel with convex and concave deformations are compared in Fig. 10. The different deformations alter the flow significantly. In the concave case, an expansion occurs as the flow encounters the left support. The fluid density decreases through the expansion but it begins to increase significantly as the flow turns through the bottom of the concave panel. Recompression occurs and a shock eminates from the right support. The boundary layer is al tered differently for the two cases as well. For concave deformation, the boundary layer thickens after the flow encounters the left support, and then it thins as the flow approaches the right support. These differences in the flow are illustrated clearly by the heating rate distributions which are compared in Fig. 11 at $t=30 \mathrm{~s}$. For the concave case, as with the convex case, there is an overall drop in heating rate due to the rise in plate temperature. However, due to the strong recompression at the right support for the concave case, there is a sharp rise in the heating rate above the flat plate value near $x=5$.
This example illustrates that flow-thermalstructural coupling can significantly alter derodynamic heating rates. Only very modest deformations occurred, but flow features were altered significantly. The location of the panel supports and the associated cases of convex and concave deformations caused the flow fields to differ considerably. Heating rate distributions changed markedly. In both cases, the simple initial boundary layer flow was altered to produce much more complex flows that can only be predicated by solving the full Navier-Stokes equations.

\section{$5^{\circ}$ Panel}

The finite element model and boundary conditions for the $5^{\circ}$ panel are shown in Fig. 12 . The model, boundary conditions and solution procedure are identical to the flat panel application. The difference is that in the undeformed state, the 5 panel presents a compression corner to the flow with a more complex initial flow, a more complex initial heating rate distribution and higher local heating rates. The structural boundary conditions for the panel are located at the lower corners causing convex deformation into the flow-field.

The effect of the panel deformation on the flow density distribution is shown in Fig. 13. Density distributions are compared for the undeformed panel and the deformed panel at $t=30 \mathrm{~s}$. For the undeformed panel a strong shock eminates from the compression corner at the left support, and the panel deformation makes the shock stronger. For the undeformed panel, an expansion occurs as the flow turns the "sharp" corner at the right support. However, the panel deformation makes the corner more rounded, and the expansion region moves upstream near the middle of the bowed panel. More significantly, for the undeformed panel there is a smali recirculation at the left support that becomes much larger as the panel deforms giving a "stronger" compression corner. The recirculation region has a major effect upon surface quantities such as skin friction and heating rate.

The heating rate distribution for the undeformed and deformed panel are compared in Fig. 14. The initial drop in heating rates at $x=1$ is associated with the boundary layer thickening and the onset of the flow separation as the flow approaches the corner. As the panel deforms, the flow separates farther upstream giving a larger recirculation region as indicated by the drop in heating rate at $x=0.4 \mathrm{in}$. for the deformed plate. As with the flat plate there is an overall drop in the heating rate distribution due to the rise in plate temperature. The panel deformation, as before, significantly alters the heating rate distribution.

If the analysis continued for longer times, the panel deformation increases, and the recirculation region grows in size until it reaches the inflow boundary of the finite element model. Then the analysis becomes invalid. Thus, this problem demonstrates that the effect of the deformation on the flow field must be given careful consideration in planning the computational domain. 


\section{Concluding Remarks}

A finite element approach for the coupling of flow, thermal and structural analyses of aerodynamically heating panels is described. The paper brings together for the first time the solution of the Navier-Stokes equation for high speed compressible flow and the solution for the associated, thermal-structural equations by a single finite element algorithm in one integrated, vectorized program.

The approach is used to study flow, thermal and structural interactions of two thin metallic panels proposed for an experimental study in the NASA Langley 8 foot high temperature tunnel. Numerical results are presented that demonstrate the effect of the panel deformation and temperature on the flow field and aerodynamic heating. For Mach 6.6 tunnel flow conditions, panel deformations alter the flow significantly. The panel deformations introduce shocks, expansions and recirculation regions in the flow. Heating rate distributions are altered significantly. For the test durations studied (up to 30 seconds) the effect of the rising pane) temperature is to lower overall heating rates. The effect of the panel deformations are to increase local heating rates on windward surfaces.

The coupling of the flow, thermal, structural analyses has provided insight into some of the fundamental features of interaction of supersonic flow with heated panels. Future analyses will study interactions on more realistic structures of current design interest such as the leading edge of the scramjet fuel injection strut.

\section{Acknowledgement}

We are pleased to acknowledge the continuing support of the Aerothermal Loads Branch at the NASA Langley Research Center and the advice and encouragement of our technical monitor, Dr. Allan R. Wieting.

\section{References}

1. Shideler, J. L., Webb, G. L. and Pittman, C. M.: "Verification Tests of Durable Thermal Protection System Concepts", Journal of Spacecraft and Rockets, Vol. 22, No. 6, Nov.Dec. 1985, pp. 598-604.

2. 01 sen, G. C. and Smith, R. E.: "Analysis of Aerothermal Loads on Spherical Dome Protuberance," AIAA Journa1, Vol, 23, No. 5, May 1985, pages 650-656.

3. Glass, C. E. and Hunt, L. R.: "Aerothermal Tests of Spherical Dome Protuberances on a Flat Plate at a Mach Number of 6.5", NASA Technical Paper 2631, Dec. 1986.
4. Bey, K. S.; Thornton, E. A.; Dechaumphai, P.; and Ramakrishnan, R.: "A New finite Element Approach for Prediction of Aerothermal LoadsProgress in Inviscid Flow Computations," presented at the AIAA 7th Computational Fluids Dynamics Conference, Cincinnati, Ohio, July 15-17, 1985. AIAA Paper No. 85-1533. Al SO NASA TM 86434.

5. Thornton, E. A. and Ramakrishnan, R.: A Finite Element Approach for Solution of the 30 Euler Equations," presented at AIAA 24 th Aerospace Scfences Meeting, Reno, Nevada, January 6-8, 1986, AIAA-86-0106.

6. Thornton, E. A.; Dechaumphai, P.; and Vermaganti, G.: "A Finite Element Approach for Prediction of Aerothermal Loads," presented at the AIAA/ASME 4th Joint Fluid Mechanics, Plasma Dynamics and Lasers Conference, May 12-14, 1986, AIAA-86-1050.

7. Thornton, E. A. and Dechaumphai, P.: "A Taylor-Galerkin Finite Element Algorithm for Transient Nonlinear Thermal-Structural Analysis," presented at the AIAA/ASME/AHS 27 th Structures, Structural Dynamics and Materials Conference, May 19-21, 1986, AIAA Paper No. 86-0911 CP.

8. Cook, R. D.: Concepts and Applications of Finite Element Analysis, John Wiley and Sons, 1984, pp. 296-297.

9. Zienkiewicz, $0 . \quad C$. and Lohner, R.: "Accelerated Relaxation or Direct Solution? Future Prospects for FEM" International Journal for Numerical Methods in Engineering, Vol. 21, pp. $1-11,1985$. 


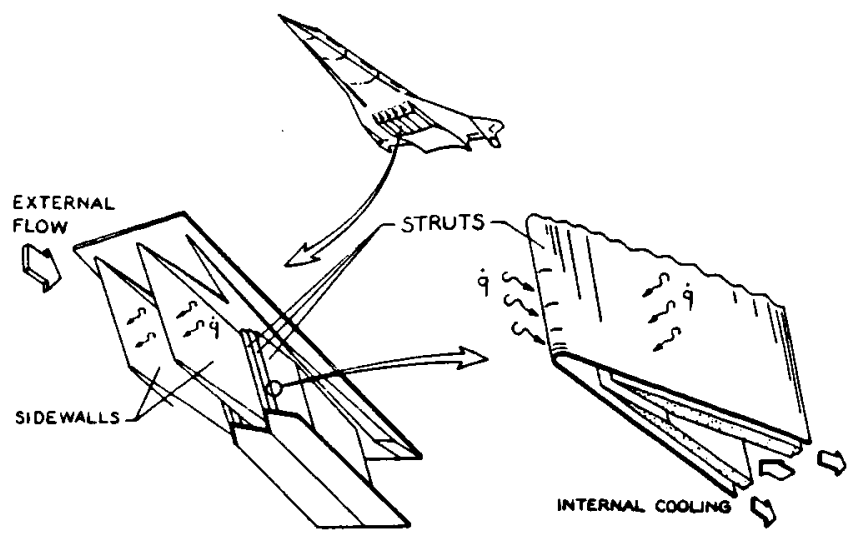

Fig. 1 - Potential areas for fluid-thermalstructural interactions on aerospace plane scramjet engine structure.

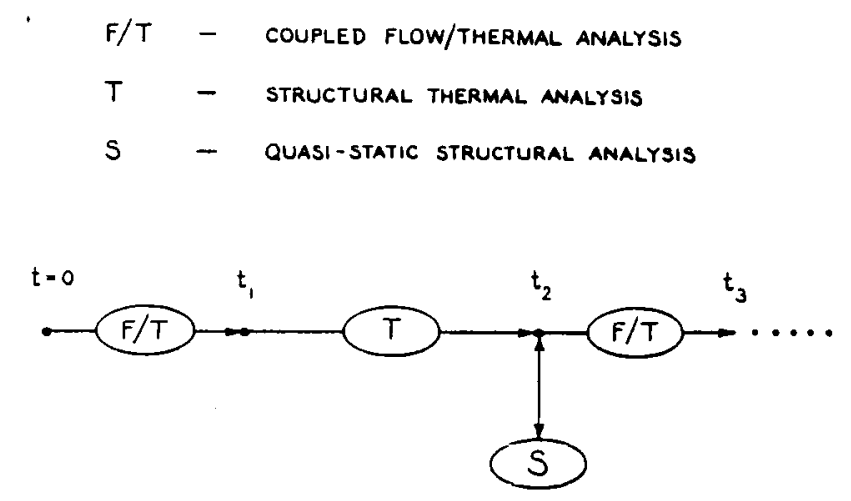

Fig. 2 - Solution sequence for coupled flowthermal-structural analysis.

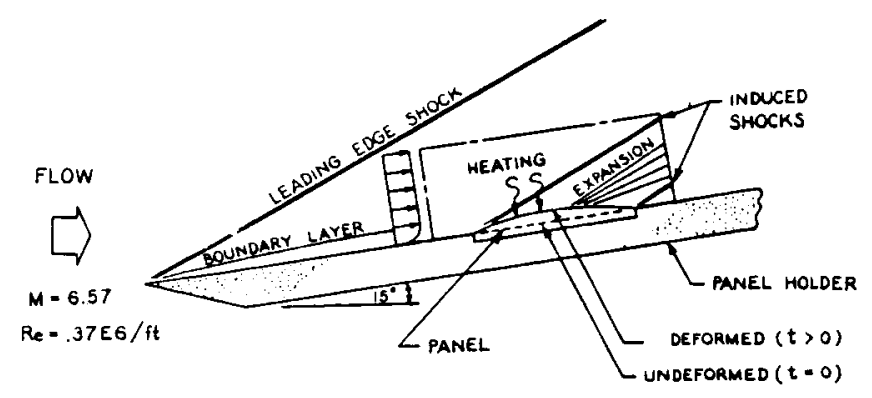

Fig. 3 - Schematic diagram of experiment to validate flow-thermal-structural analysis.
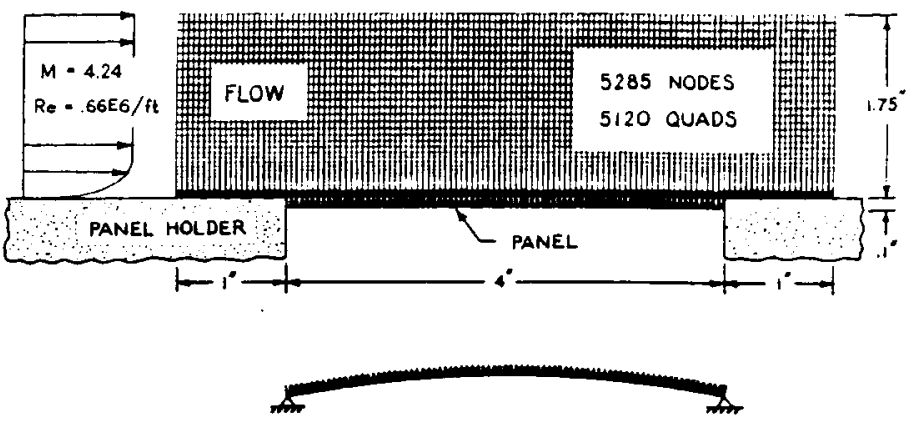

PANEL WITH CONVEX DEFORMATION

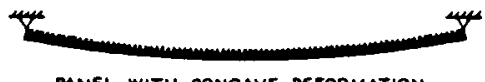

Fig. 4 - Coupled flow-thermal-structural finite element model and boundary conditions for flow over flat plate.

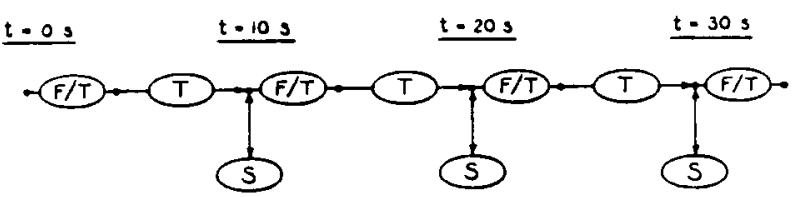

Fig. 5 - Solution sequence for coupled flowthermal-structural analysis of flow over flat plate.

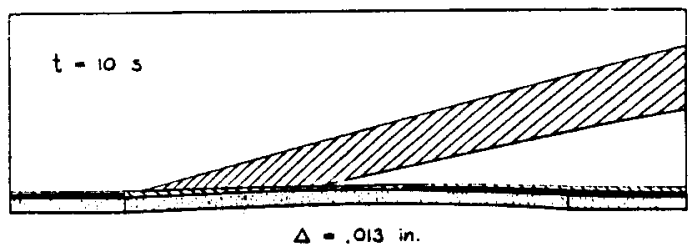

$\rho=10^{-8}, 1 b_{f}-3^{2} / 10^{4}$
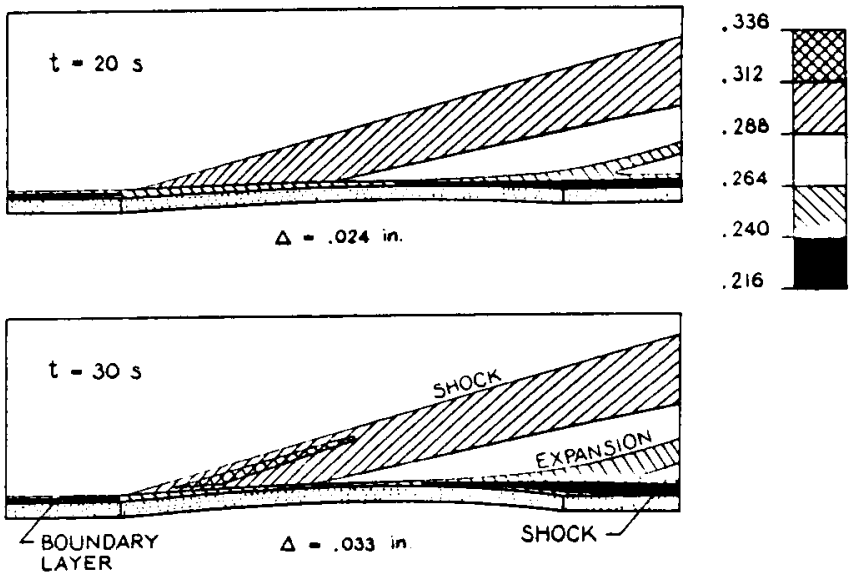

Fig. 6 - Flow density distributions for panel with convex deformation. 


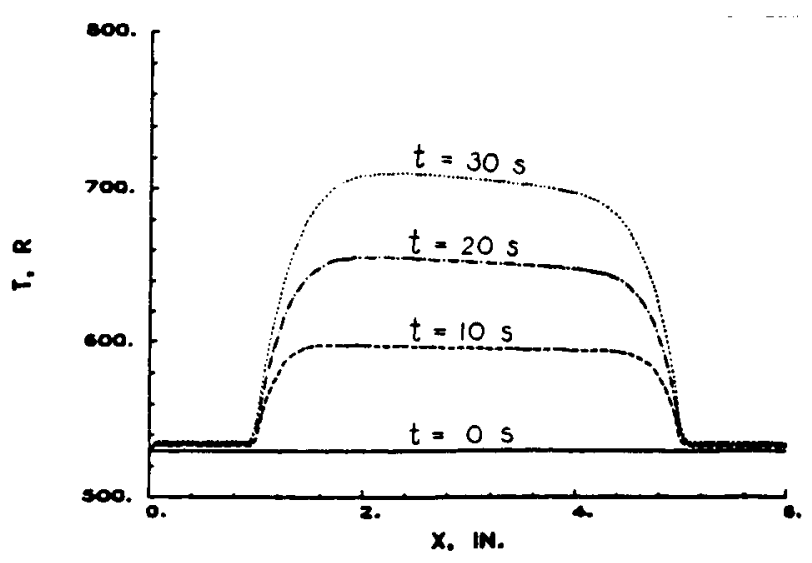

Fig. 7 - Temperature distributions at fluidpanel interface for panel wi th convex deformation.

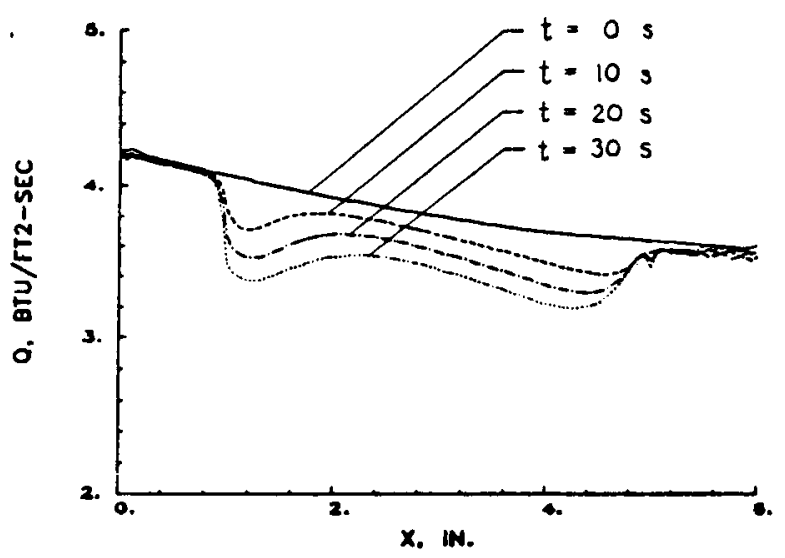

(a) Evolution of heating rate distribution

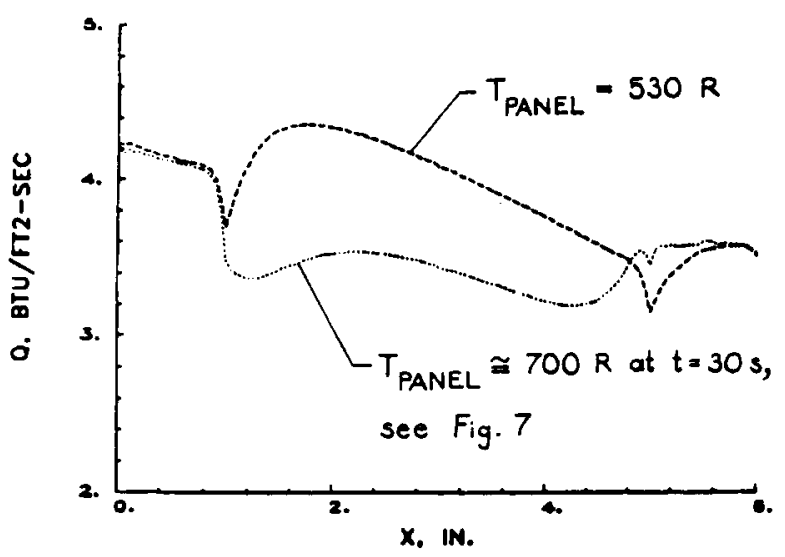

(b) Effect of panel temperature on heating rate distribution

Fig. 8 - Heating rate distributions for pane? with convex deformation.

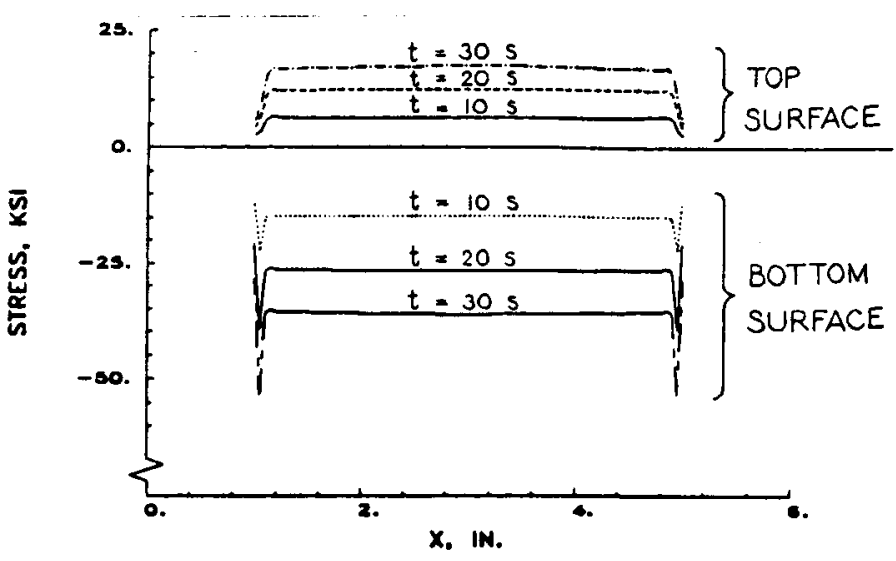

Fig. 9 - Thermal stress distributions for panel with convex deformation.

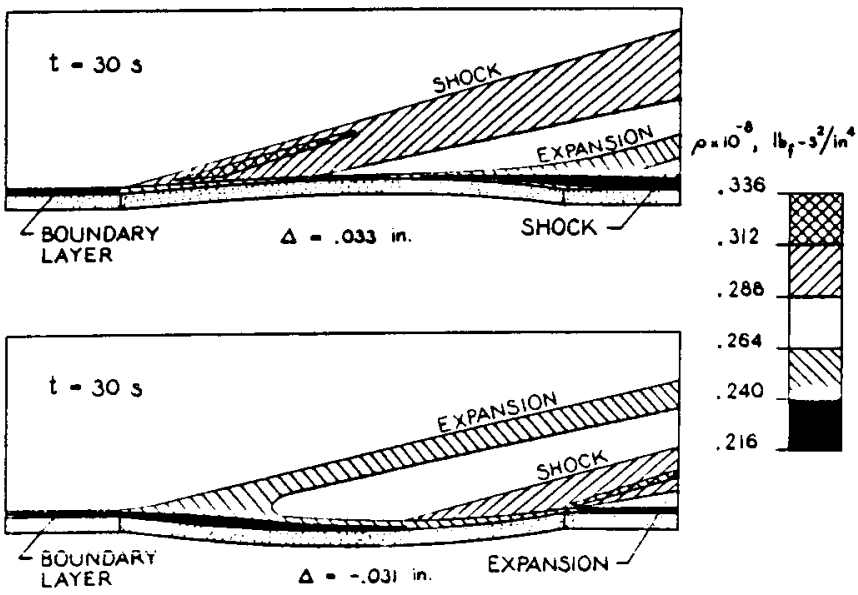

Fig. 10 - Flow density distributions for panel with convex and concave deformations at 30 seconds.

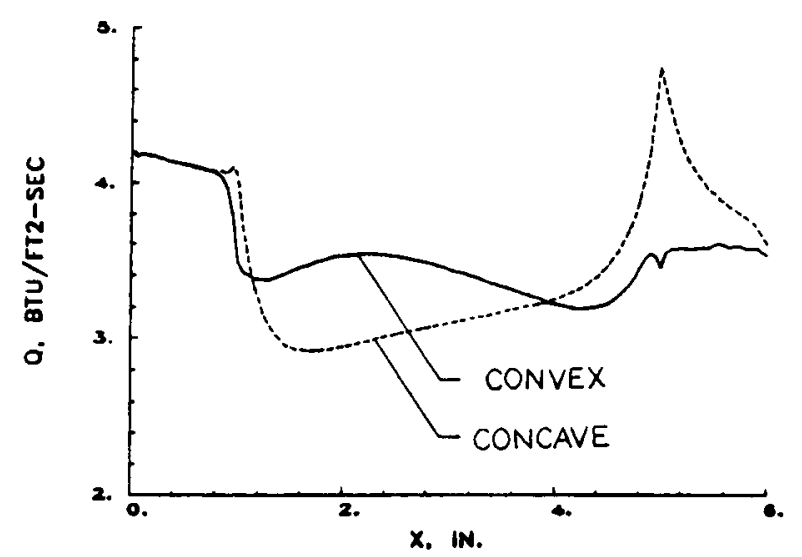

Fig. 11 - Heating rate distributions for panel with convex and concave deformations at 30 seconds. 


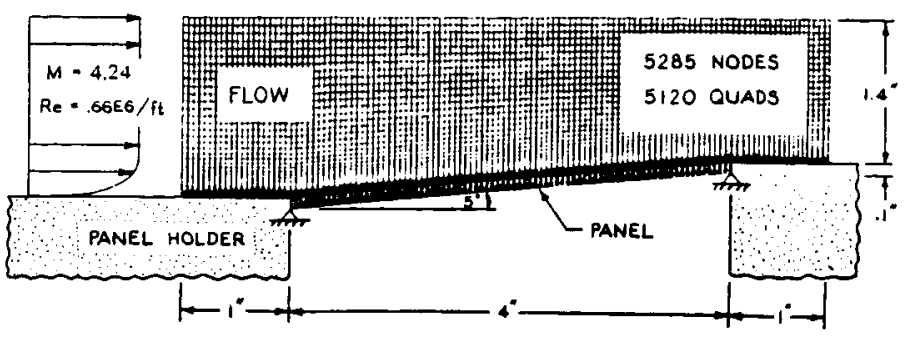

Fig. 12 - Coupled flow-thermal-structural finite element model and boundary conditions for flow over $5^{\circ}$ panel.

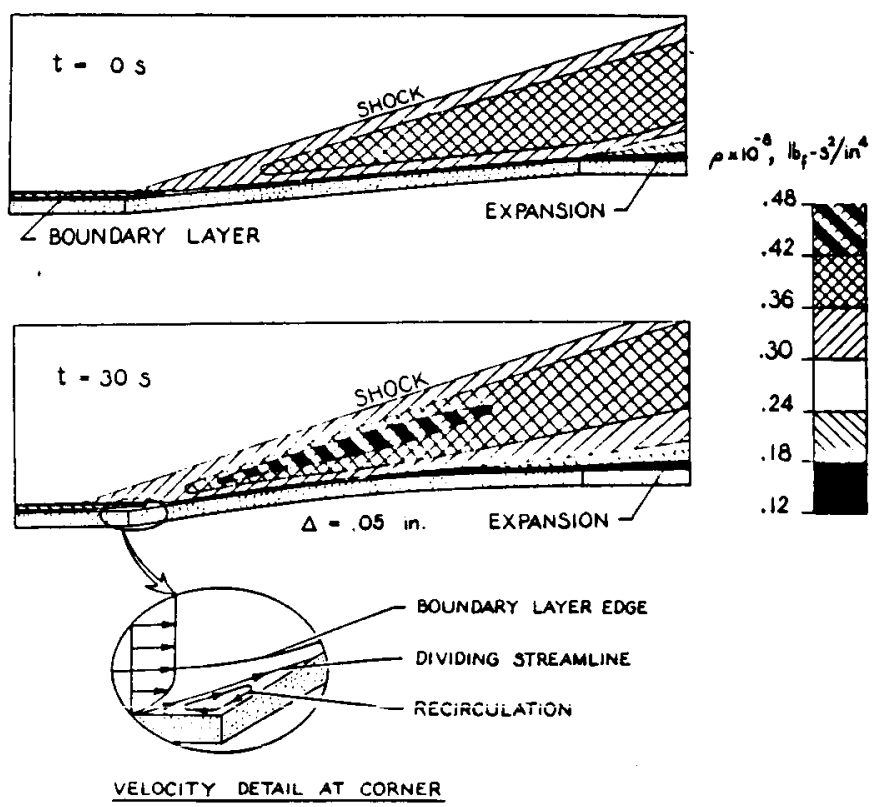

\section{ORIGINAE PAGE IS} OF POOR QUAIJT'Y

Fig. 13 - Flow density distributions for $5^{\circ}$ panel.

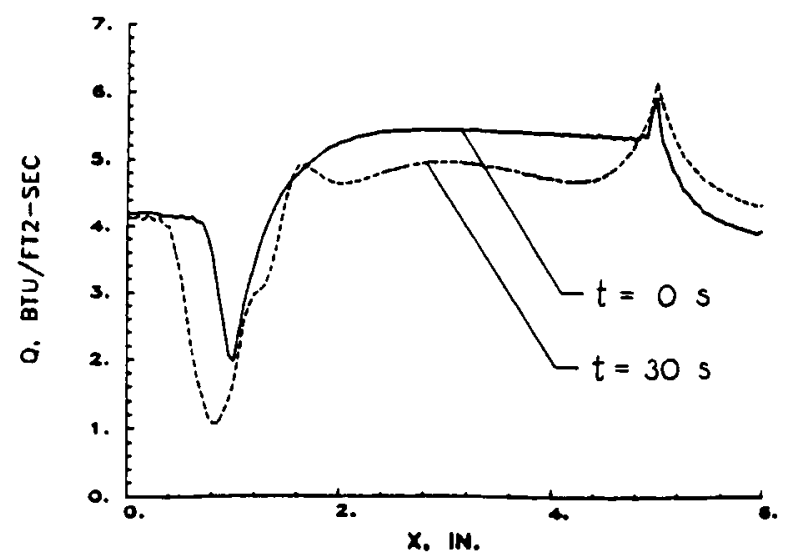

Fig. 14 - Heating rate distributions for $5^{\circ}$ panel. 